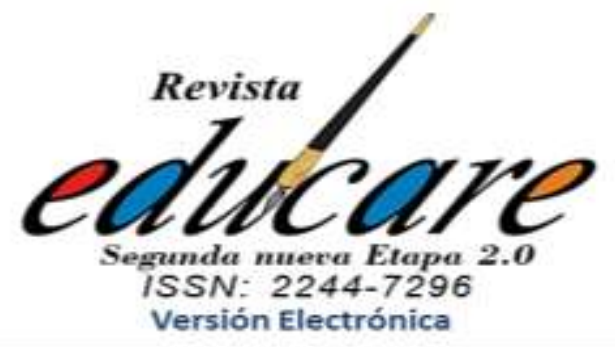

Volumen 25 NNo $^{\circ}$ Enero - Abril 2021

(201-223)

Antiche Valera, Johnny Ramón *

ORCIID: https://orcid.org/0000-0002-0662-8021

Universidad Pedagógica Experimental Libertador-

(Venezuela)

Piñero Martín, María Lourdes **

ORCID: http:// orcid.org/0000-0002-7038-2871

Universidad Pedagógica Experimental Libertador(Venezuela)

Vanga Arvelo, María Giuseppina ***

ORCID: https://orcid.org/0000-0003-0143-8381

Universidad Técnica de Manabí

( Ecuador)

Sáenz Gavilanes, Jessica Vicenta **

ORCIID: https://orcid.org/0000-0002-9647-6657 Universidad Laica Eloy Alfaro de Manabí (Ecuador)

Carmen Auxiliadora, Lucas Mantuano

ORCID: https://orcid.org/0000-0002-4287-2902

Universidad Laica Eloy Alfaro de Manabí

(Ecuador)

*profesor de mecánica industrial en la Universidad Pedagógica Experimental LibertadorInstituto Pedagógico de Barquisimeto (Venezuela), es Magister en Educación Técnica por la Universidad Pedagógica Experimental Libertador- Instituto Pedagógico de Barquisimeto(Venezuela); es Doctor en Educación johnnyantiche@gmail.com

** Lic. En Educación, mención Ciencias Sociales en la Universidad del Zulia (Venezuela), continuó estudios de cuarto nivel en la Universidad del Zulia como Mg.Sc. en Planificación y Gerencia de la Ciencia y Tecnología, y en la Universidad Yacambú (Venezuela) como Mg. en Gerencia Educativa. Es Doctora en Ciencias mención Investigación Malopima11@gmail.com;

*** Especialista en Formación Docente y Programación Neurolingüística; es Magíster en Gerencia Empresarial por la Universidad Rafael Belloso Chacín(Venezuela); es Doctor en Arquitectura mgvanga@yahoo.com

**** Magister en Docencia e Investigación Educativa en la Universidad Técnica de Manabí, actualmente cursando Doctorado en Ciencias de la Educación Superior jessikvsg@gmail.com

***** Magister en Tributación y Finanzas en la Universidad de Guayaquil, actualmente cursando Doctorado en Ciencias Económicas en la Universidad del Zulia-Venezuela. Profesor adscrito a la Facultad de Hotelería y Turismo de la Universidad Laica Eloy Alfaro de Manabí carmen.lucas@uleam.edu.ec

\section{ACCIÓN PEDAGÓGICA PARA LA INNOVACIÓN EN LA EDUCACIÓN TÉCNICA INDUSTRIAL: UNA APROXIMACIÓN ETNOGRÁFICA}

\author{
PEDAGOGICAL ACTION FOR INNOVATION IN \\ INDUSTRIAL TECHNICAL EDUCATION: AN \\ ETHNOGRAPHIC APPROACH
}

\section{Recibido: \\ 04-12-2020 \\ Aceptado: \\ 28-03-2021}



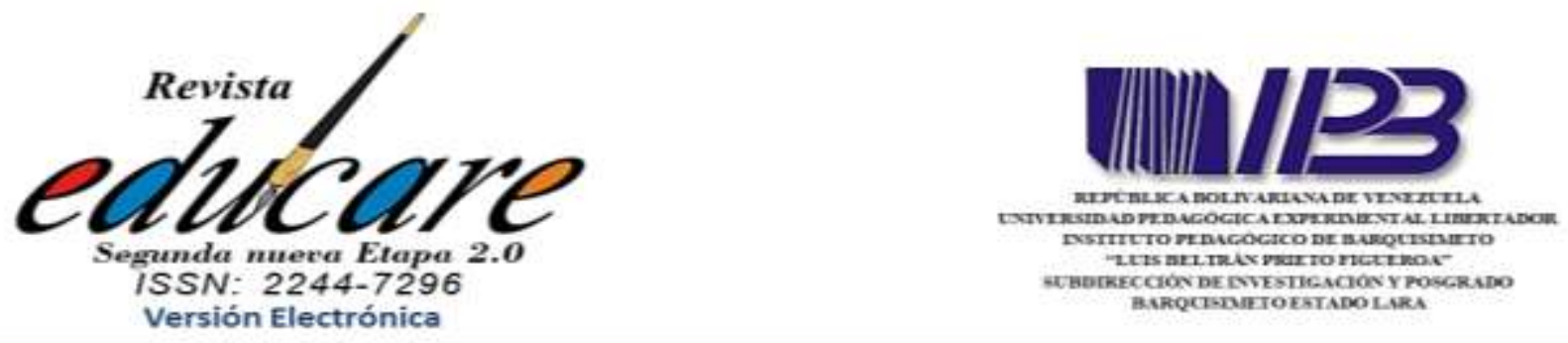

\section{Resumen}

El objetivo fue interpretar el significado de la práctica pedagógica que los docentes realizan para llevar a cabo una educación técnica industrial orientada hacia la innovación, en el contexto de la Escuela Técnica Industrial "La Carucieña" en la ciudad de Barquisimeto, Venezuela. Se empleó el método etnográfico interpretativo, en la perspectiva metodológica cualitativa; se utilizó la observación participante en las aulas y talleres, y la entrevista en profundidad a cinco docentes. El procedimiento de codificación y categorización generó como resultado dos categorías orientadoras: (a) La innovación en el acto pedagógico de las Escuelas Técnicas Industriales, y (b) Pedagogía del aprendizaje para la innovación, a las cuales se realizó el proceso hermenéutico. Como reflexión final se tiene que la esencia del Ser innovador en el acto pedagógico es el docente, pues sus estudiantes serán el reflejo de la actitud hacia la necesidad de mejorar, de incorporar cambios y de la dedicación a sobrepasar la educación de la rutina y la inercia.

Palabras clave: Acción pedagógica; educación técnica industrial; etnografía; innovación.

\section{Abstract}

Abstract: The objective was to interpret the meaning of the pedagogical practice that teachers carry out to carry out an industrial technical education oriented toward innovation, in the context of the Industrial Technical School "La Carucieña" in the city of Barquisimeto, Venezuela. The interpretive ethnographic method was used in the qualitative methodological perspective; participant observation was used in classrooms and workshops, and the in-depth interview of five teachers was used. The coding and categorization procedure resulted in two guiding categories: (a) Innovation in the pedagogical act of the Industrial Technical Schools, and (b) Pedagogy of Learning for Innovation, to which the hermeneutic process was carried out. As a final reflection it is necessary that the essence of the innovative being in the pedagogical act is the teacher, because his students will be the reflection of the attitude towards the need to improve, to incorporate changes and the dedication to overcome the education of routine and inertia.

Keywords: Pedagogical action; industrial technical education; ethnography; innovation 
La educación representa para todas las sociedades del mundo, la auténtica esencia en la búsqueda del mejoramiento continuo, como una oportunidad para articular las estructuras del conocimiento, que permitan asumir retos y propiciar progreso, desarrollo y bienestar en los ciudadanos. Al mismo tiempo, una de las modalidades educativas que aspira ser factor de innovación, productividad, cambio para el desarrollo y bienestar socioeconómico de la población, es la educación técnica. Es por ello que, desde hace varios años en América Latina, específicamente esta modalidad educativa, busca ampliar su función, en el sentido de que no solo se debe trabajar para educar a los estudiantes, sino también se debe incidir en el desarrollo de las familias y de su entorno.

Desde esta perspectiva, las escuelas técnicas pueden representar una función mediadora en aras de constituirse en el espacio para el encuentro afectivo, solidario, de confianza y de innovación, que favorezca la conformación relaciones sociales de cooperación, al tiempo que propicie los medios provechosos y atractivos para el aprendizaje creativo, a los fines de enfatizar la formación para el trabajo como fuerza generadora del desarrollo que necesitan las naciones.

Además, se debe tener presente las preocupaciones del mundo productivo, en materia de innovación, tecnología y servicios, que tienden con insistencia a la búsqueda globalizante de alternativas educativas, enriquecidas con un componente humanizador y ecológico, sustituyéndose progresivamente la simple visión productiva de las organizaciones por otra visión de mayor contenido social y humano, lo que no excluye y menos deslinda al proceso educativo técnico.

Esta visión se contrapone a la postura economicista, donde la formación para el trabajo responde a intereses productivos y replicadores de una estructura predominante con base en la empleabilidad socio económica, la cual puede verse reflejada en la afirmación de Calderón(2002), al indicar que:

En la educación para el trabajo se ubicaría las estrategias sociales dirigidas a solventar las desigualdades económicas de la población. Para ello, se espera que los nuevos trabajadores sean personas que permanentemente desarrollan habilidades técnicas y profesionales que los hacen empleables y que son capaces de agregar valor en su 
trabajo. Gente con más habilidad intelectual, comunicacional y de trabajo en equipo.

Trabajadores que se ganan el pan con el sudor de sus mentes. (p33)

Sin embargo, no se trata de formar trabajadores por formarlos, para que estén únicamente capacitados de conseguir un empleo y obtener una buena remuneración; por el contrario, es necesario que exista un proceso integrador donde se conjuguen la competencia para desarrollar un oficio, junto a la vocación humanizadora (conciencia del ser) y social (para una conciencia con el otro). Este tipo de perspectiva que orienta la formación para el trabajo, que aún hoy en día persiste, pareciera caracterizarse por defender el aumento de la riqueza material a costa de la riqueza humana. No obstante, el crecimiento material, ni aun si resultare sostenible sería insuficiente, si éste no va incorporando e impregnado la calidad humana, en virtud que la esencia del desarrollo debe consistir en que todos los seres humanos vivan mejor. Es decir, la planificación del desarrollo debe consistir en el progreso integral del ser para toda la vida.

En el contexto de tal planteamiento, desde la última década del siglo $\mathrm{XX}$, tal como señala Simone (1993) ya se observaba la necesidad de configurar en la formación para el trabajo y en la formación técnica, un espacio que conllevase al aprendizaje permanente, basado entre otros aspectos en un amplio acceso a la información, al análisis y a la interacción con el entorno económico y social, sumado a un aprendizaje innovador, sustentado en la capacitación, la integración y la autodidaxia. Todo ello se potenciaría si estimulan valores personales como la determinación, la perseverancia, la curiosidad o la creatividad en los procesos lógicos y analíticos, con lo cual se posibilitaría participación, la reflexión, la crítica y la Innovación.

Es así como organizaciones pioneras en esta modalidad educativa como la Federación Internacional Fe y Alegría (1995), viene planteando también a finales del siglo XX que, aun cuando la educación está orientada fundamentalmente hacia la capacitación humana, laboral y política que genere riqueza y garantice su equitativa distribución, "la educación en y para el trabajo, implica construir un diseño educativo que ofrezca a los estudiantes oportunidades de crear y de producir cambios, que les permita ganar en la sociedad espacios de participación real para así poder transformarla" (Fe y Alegría,1995). Veinte años más tarde y en pleno siglo 
XXI, esta aspiración continua cobrando vigencia apreciándose en la Declaración de Incheon en el Foro Mundial sobre la Educación entre otros aspectos lo siguiente:

Nuestra visión es transformar las vidas mediante la educación, reconociendo el importante papel que desempeña la educación como motor principal del desarrollo y para la consecución de los demás ODS propuestos. (Organización de las Naciones Unidas para la Educación, la Ciencia y la Cultura, UNESCO, 2015, p. iii)

(...) Reconocemos que la educación es clave para lograr el pleno empleo y la erradicación de la pobreza. Centraremos nuestros esfuerzos en el acceso, la equidad, la inclusión, la calidad y los resultados del aprendizaje, dentro de un enfoque del aprendizaje a lo largo de toda la vida. (UNESCO, 2015, p.iv)

Esta demanda también quedó plasmada en el documento Agenda 2030, al señalar en el punto 4 (Naciones Unidas, 2018) que "la consecución de una educación de calidad es la base para mejorar la vida de las personas y el desarrollo sostenible"; y por lo tanto es coherente con la Meta 4.4. en la que se precisa: "De aquí a 2030, aumentar considerablemente el número de jóvenes y adultos que tienen las competencias necesarias, en particular técnicas y profesionales, para acceder al empleo, el trabajo decente y el emprendimiento"(Naciones Unidas, 2018, p. 28).En tal sentido, puede apreciarse que los propósitos de la educación técnica "con independencia de los factores históricos, situaciones económicas y tradiciones políticas que condicionan su implementación en los países, pueden ser enunciados al menos desde tres perspectivas: económica, social y de sustentabilidad" (Sevilla. 2017;p.12).

Es por ello que emerge desde esta visión, la necesidad de que las instituciones educativas, tanto formales como no formales dedicadas a la formación para el trabajo, "proporcionen a sus estudiantes una sólida formación científico-técnica general, que desarrolle sus destrezas intelectuales, de modo que sean capaces de razonar, proponer e innovar" (Simone, 1993). Por lo que sus conocimientos, valores, actitudes, habilidades, competencias y destrezas deben ser útiles para superarse individualmente y contribuir al desarrollo colectivo y transformación de su entorno educativo, familiar y social.

La vinculación de la educación e innovación como vía para el desarrollo y la transformación social, es un planteamiento que han argumentado algunos autores y organismos internacionales como: Drucker (2000); Blanco (2005); Robalino \& Eroles 
(2010); Ibarrola (2010); Martín-Gordillo \& Castro-Martínez (2014); Cobo (2016); UNESCO (2016), entre otros. En los discursos cotidianos y académicos, es apreciable que dimensiones como innovación educativa, educación innovadora, educar para innovación, innovar para aprender, escuelas innovadoras, entre otras, están incorporadas al hacer referencia a los retos y desafíos de la educación en todos sus niveles y modalidades, desde su rol transformador. Así lo afirman Martín-Gordillo \& Castro-Martínez (2014): "Entre la innovación y la educación hay un vínculo muy estrecho, casi de naturaleza antropológica. La tarea educativa supone transmitir a los niños y jóvenes las herramientas necesarias para su incorporación a la vida activa en la sociedad" (p.5); de allí, "Que hay que innovar (más) para educar (mejor) es una idea comúnmente admitida desde hace tiempo y una demanda clara hacia los sistemas educativos y hacia los docentes. Pero la innovación no es solo un medio educativo, sino quizá el fin principal de la educación" (Martín-Gordillo \& Castro-Martínez ;2014, p.5).

Al hacer referencia a la innovación como un acto vinculado al proceso de aprender, la UNESCO (2016) plantea lo siguiente:

La innovación está fundamentada sobre el aprendizaje, en cuanto ésta se encuentra ligado a la acción transformadora del mundo, en tanto tiene un profundo sentido de cambio, pues produce unas características que no se dan por generación espontánea, ya que éstas deben ser organizadas y planificadas para que el espacio de innovación aprendizaje logre sus impactos en los múltiples ámbitos de la sociedad.

La organización del aprendizaje vinculado a la innovación, implica pues, una acción pedagógica que se aleja de las formas tradicionales de enseñar y de guiar el aprendizaje, lo cual implica según Pomares et.al. (2020) "la introducción de cambios en el proceso enseñanza aprendizaje que tributen al mejoramiento de su calidad, asumiendo un carácter progresivo o radical" (p.95)

En tal sentido, la Cumbre Mundial para la Innovación en Educación de 2014 (Fundación Codespa, 2014), declara que "la educación en las aulas va a tender a un nuevo modelo pedagógico en el que, además del conocimiento, se van a desarrollar habilidades hasta ahora olvidadas por la escuela como la innovación, la creatividad, la inteligencia existencial, el emprendimiento social, el liderazgo existencial, la colaboración, la comprensión y la empatía, entre otros". Y ante este nuevo escenario pedagógico los 
profesores tienen que salir de su zona de confort, luchar contra sus inseguridades y cambiar hábitos y costumbres. De allí que:

Toda innovación supone entrar en el terreno de lo desconocido, y esto implica ciertas dosis de riesgo, incertidumbres y conflictos. No hay aprendizaje sin desequilibrio y ni conflicto cognitivo. Cuando los docentes intentan probar nuevas ideas o desarrollar nuevas prácticas, existe un momento inicial de desequilibrio y confusión debido a la confrontación entre lo nuevo y lo viejo" (Mogollón, 2015, p.13).

Por ello según Cobo (2016), hay una necesidad de:

(...) re-perfilar el papel de los docentes bajo una relación diferente con el conocimiento. Una relación distinta a la que tradicionalmente habían tenido (y bajo la cual muchos fuimos formados). Esto significa que el valor central ya no está en el acceso o dominio de un conocimiento en particular sino en la capacidad de desfragmentar y reconstruir conocimientos bajo nuevas combinaciones, formatos y canales. Una re-significación delo que implica ofrecer y recibir educación" (p.10).

Se entiende entonces, que una acción pedagógica que pretenda vincular el aprendizaje para una educación en la innovación, no es posible sin una actuación docente que oriente la transformación, la creación y recreación del mundo, pues a decir de la UNESCO "la innovación no es una simple mejora sino una transformación; una ruptura con los esquemas y la cultura vigentes en las escuelas" (2016, p. 14). Tales requerimientos son especialmente necesarios en los espacios pedagógicos que orientan la educación técnica profesional, y concretamente en el área industrial, la cual está impregnada de ciertas especificidades y claras innovaciones que se distancian de la modalidad regular escolar.

Entre las distinciones de la educación técnica industrial, Ibarrola (2010) señala las siguientes: a) se define por referencia directa de los sectores económicos y las ramas ocupacionales y de preferencia ocupaciones puntuales; b) predomina la intención de formar directamente para el desempeño laboral; c) privilegia la formación práctica, centrada en el ejercicio del trabajo mismo o, particularmente, en la manipulación de los equipos y tecnología que lo constituyen; d) modifica por lo mismo, el uso del tiempo y genera espacios de enseñanza diferentes al aula, en particular los talleres y los procesos productivos directos.

Es menester que, en las escuelas técnicas, la acción pedagógica exista en un ambiente 
apropiado para la innovación, donde se puedan establecer la formación de habilidades y destrezas, que coadyuven la configuración de acciones para la adquisición de conocimientos y prácticas a los fines de sustentar aquellas transformaciones que optimicen las actividades educativas. En este contexto es posible incrementar aquellos valores, hábitos, aptitudes y conductas vinculadas a las competencias necesarias que demandan el contexto empresaria, social y académico a los estudiantes que aspiran ingresar al campo laboral o continuar con sus estudios universitarios. De modo pues, que la innovación en el aula "desde una visión sistémica, se traduce en una mirada más holística, que integra las características de los sujetos y de las metodologías como parte de los factores asociados al proceso de enseñanza aprendizaje y sus resultados, entre otros aspectos" (Maldonado-Fuentes \& RodríguezAlveal,2016).

Por ello, una característica fundamental de esta nueva concepción de la formación, radica en la incorporación de contenidos y metodologías propios de lo que se ha dado en llamar: Educación Tecnológica, como lo expresa la Organización Internacional del Trabajo que consiste en:

(...) en registrar, sistematizar, comprender y utilizar el concepto de tecnología, histórica y socialmente construido, para hacer de él un elemento de enseñanza, investigación y extensión, en una dimensión que exceda los límites de las simples aplicaciones técnicas: como instrumento de innovación y transformación de las actividades económicas, en beneficio del hombre como trabajador y del país. (OIT, 2001,p.36)

Al focalizar la mirada en Venezuela, se puede precisar que según el artículo 25 de la Ley Orgánica de Educación, el nivel de educación media establece dos opciones: educación media general con duración de cinco años (de primero a quinto año), y educación media técnica con duración de seis años (Asamblea Nacional de la República Bolivariana de Venezuela, 2009).De acuerdo a este instrumento legal, la enseñanza en la educación media técnica venezolana comprende la formación de bachilleres y de técnicos medios en diversas especialidades de las áreas industriales (ETI), comerciales (ETC), agropecuarias (ETA) y asistenciales. 
De acuerdo a Antiche (2012,p.77) este proyecto apuntaba hacia la formación de un ciudadano con niveles adecuados para asumir el reto de los sectores productivos de un país que entraba en el llamado "socialismo del siglo XXI". Con ello se buscó apoyar el emprendimiento de las áreas estratégicas de la nación venezolana, al tiempo de lograr la inserción y profundización de la formación de la población joven mediante el valor del trabajo; de esta manera se buscaba alcanzar un aporte sólido a la "Revolución Productiva" en concordancia con la adecuación del aparato productivo a los nuevos lineamientos político económicos del entonces gobierno del Hugo Chávez Frías.

A pesar de los ambiciosos objetivos educativos que desde el punto de vista de política educativa significó la creación de esta modalidad educativa, según Ramírez \& León (2012),el bachillerato académico es la modalidad dominante, pues ofrece mayor status social, mientras que la técnica profesional es considerada una educación para personas de escasos recursos, o para personas menos capaces. Esta situación también tiene sus implicaciones en la cultura académica, debido a que los modos pedagógicos dentro de las escuelas la convierten en lo legítimo y deseable, propiciando la hegemonía cultural del trabajo intelectual. Los referidos autores expresan que, en el ámbito de las escuelas técnicas venezolanas:

(...) se piensa que sólo son prácticas manuales y se ignora la necesidad de enseñanzas científicas y tecnológicas. Es común la deficiente preparación de los estudiantes en el núcleo común o área académica, con relación a las bases científicas indispensables para el área técnica, que impiden la debida correspondencia entre teoría y práctica" (Ramírez \& León; 2012,p.35).

Es por ello que en algunos estudios como el de Cuello (2006), venían advirtiendo desde hace más de una década que en las ETI venezolanas prevalece una indisposición a la innovación tecnológica y a la aplicación práctica de la creatividad, evidenciada en el poco esfuerzo que realizan los docentes y estudiantes en el mejoramiento y modificación de sus equipos, maquinarias y procesos productivos. También se muestra indiferencia hacia la promoción y estimulación de la creatividad y la innovación tecnológica, ya que se mantienen alejados de los programas y actividades convocadas por el Ministerio de Ciencia y Tecnología (MCT) y otras organizaciones científicas.

En el contexto actual venezolano, caracterizado por la agudización de los problemas de pobreza, aumento de los índices de desempleo, deserción escolar, cierre de empresas, y 
donde se ha marcado la brecha existencial entre la educación para el trabajo y la demanda del mercado laboral, la Escuela Técnica Industrial "La Carucieña" en el Estado Lara, funciona en la zona oeste de la ciudad de Barquisimeto, en uno de los sectores de mayor concentración de población de los estratos socioeconómicos más bajos. Es del interés de este estudio etnográfico develar e interpretar el significado de la práctica pedagógica que los docentes realizan para llevar a cabo una educación técnica industrial basada en los principios de la innovación, en la esperanza de crear y mantener un ambiente de aprendizaje que motive a la prosecución de una población estudiantil duramente afectada por la situación económica que caracteriza la sociedad venezolana de los últimos años. En este sentido, la investigación se enmarca en la perspectiva metodológica cualitativa, específicamente guiada por el Método Etnográfico.

\section{Sobre el Método}

Con respecto al método de investigación, se optó por una de las alternativas metodológicas que ofrece la rigurosidad científica de las ciencias sociales, como es la tradición etnográfica-hermenéutica o etnografía interpretativa. Al respecto, Geertz (1992) refiere que la descripción etnográfica presenta entre sus rasgos característicos: “(...) es interpretativa, lo que interpreta es el flujo del discurso social y la interpretación consiste en tratar de rescatar 'lo dicho' en ese discurso de sus ocasiones perecederas y fijarlo en términos susceptibles de consulta" (p.32).

A tal efecto, en el plano educativo, el método etnográfico interpretativo se centra en develar lo que sucede cotidianamente en los ambientes escolares, aportando testimonios significativos de la forma más descriptiva posible para luego interpretarlos y comprenderlos, y si es posible, intervenir adecuadamente en los escenarios escolares. El método se estructuró en las tres fases propuestas por Velasco \& Díaz (2006), las cuales se diferencian en: acciones, objetos y transformaciones, cuyas actividades procedimentales se especifican en la Tabla 1:

Tabla 1:Fases y acciones del Método Etnográfico Interpretativo del presente estudio

\begin{tabular}{c|cc}
\multicolumn{1}{c|}{$\begin{array}{c}\text { Fases del } \\
\text { Método }\end{array}$} & \multicolumn{1}{c}{ Descripción de las actividades implícitas desarrolladas } \\
\hline \multirow{2}{*}{ Acciones } & a) & Concepción del Proyecto \\
\hline & b) & Configuración y registros del diario de campo \\
\hline & c) & Descripción y contextualización del contexto de estudio \\
\hline & d) & Visitas y observaciones en el contexto de estudio \\
\hline
\end{tabular}




\begin{tabular}{c|lll}
\hline \multirow{4}{*}{ Objetos } & e) & Participación en las rutinas de clase y prácticas de talleres. \\
& f) & Encuentros dialógicos con los docentes \\
\hline & g) & Visitas finales. Revisión y validación de los textos transcritos \\
& de las entrevistas y fotos de observaciones a los docentes \\
\hline & h) & Contrastación de la información con los coinvestigadores \\
\hline & a) & Primeras inquietudes investigativas \\
\hline & b) & Revisión del estado del arte sobre la temática \\
\hline & c) & Indagación y reflexión epistemológica sobre el método y las \\
intenciones investigativas
\end{tabular}

Nota: Elaboración propia con datos de la investigación.

El diseño procedimental que orientó la investigación, estuvo guiado por la perspectiva metodológica cualitativa, entendida por Piñero et.al.(2020) como la dinámica interactiva de construcción y reconstrucción de del conjunto de estrategias técnico-operativas realizadas por los investigadores de este trabajo, desde la sustentación de la rigurosidad del método etnográfico elegido y "guiado mediante la reflexión epistémica constante que implica el involucramiento en el contexto social, a los fines de recrear, analizar y comprender o transformar las significaciones cotidianas del fenómeno en estudio por parte de los informantes participantes" (p.49).

En este artículo, se reportan los hallazgos devenidos de la entrevista en profundidad y observaciones participativas en las aulas de clase y talleres de prácticas industriales. Igualmente, para la elección de informantes (aquí llamados versionantes), se consideró como criterio que fuesen parte del personal docente ordinario o contratado con experiencia superior a los cinco años de antigüedad en la institución educativa objeto de estudio, los cuales se especifican en la tabla 2. 
Tabla 2: Versionantes entrevistados y la nomenclatura de los protocolos de registro

\begin{tabular}{c|c|l|l|l}
\hline Nomenclatura & Identificación & Cargo & Estudio Realizados & $\begin{array}{l}\text { Años de } \\
\text { Servicio }\end{array}$ \\
\hline V01 & RM & $\begin{array}{l}\text { Profesor jefe de los talleres } \\
\text { de mecánica }\end{array}$ & $\begin{array}{l}\text { Magíster en educación } \\
\text { técnica }\end{array}$ & 11 años \\
\hline V02 & MO & $\begin{array}{l}\text { Profesor del taller de } \\
\text { soldadura }\end{array}$ & $\begin{array}{l}\text { Magíster en educación } \\
\text { técnica }\end{array}$ & 10 años \\
\hline V03 & YM & $\begin{array}{l}\text { Profesor del taller de ajuste } \\
\text { y limado }\end{array}$ & $\begin{array}{l}\text { Profesor de mecánica } \\
\text { industrial }\end{array}$ & 09 años \\
\hline V04 & CR & $\begin{array}{l}\text { Profesor del taller de } \\
\text { soldadura }\end{array}$ & $\begin{array}{l}\text { Magíster en educación } \\
\text { técnica }\end{array}$ & 08 años \\
\hline V05 & JA & $\begin{array}{l}\text { Profesor de mecánica } \\
\text { industrial }\end{array}$ & $\begin{array}{l}\text { Magister en Educación } \\
\text { Técnica }\end{array}$ & 10 Años \\
\hline
\end{tabular}

Nota: elaboración propia con datos de la investigación.

Para la observación participante, se empleó el diario de campo, video grabadora y cámara fotográfica; mientras que para la entrevista se empleó la modalidad no estructurada, con un guion de inquietudes claves de planteamientos generadores y apoyados con una libreta de notas, lápiz y grabadora digital de audio, procesados con el programa de edición de Sound Forge (versión 7.0) antes de la transcripción con el procesador de textos. Para la transcripción, se utilizaron modelos de protocolos de registros identificados por versionante (V01,V02,V03,V04y V05),y por líneas de transcripción (L); organizados según recomendación de Piñero,et.al. (2020). Una vez transcrita la información, se procedió a la codificación y categorización del texto, como paso clave en el proceso de interpretación o hermenéusis, utilizándose la técnica denominada por los referidos autores como cromatización, y pautada en cuatro fases (Piñero et.al; 2020, p.204).

Con respecto a la legitimidad científica, se consideraron los criterios de singularidad del objeto de estudio, el consentimiento informado de los versionantes, y la triangulación. En este último caso, se aplicó la triangulación de fuentes, triangulación teórica y triangulación de investigadores (donde participaron los autores del presente artículo).

Cabe destacar, que la información recolectada fue bastante densa, siendo que la contrastación y saturación de fuentes resultó elevada para cada categoría. De allí que, por razones de espacio, los investigadores eligieron las frases o narrativas testimoniales más significativas encontradas. 


\section{Hallazgos}

Develar la acción pedagógica que los docentes realizan en la ETI La Carucieña para llevar a cabo una educación orientada hacia la innovación, implicó conocer el entendimiento al respecto desde las voces de los versionantes, y las observaciones específicas e inespecíficas registradas durante el transitar investigativo. En la tabla 3, es apreciable la significación de la categoría medular configurada como: Acción pedagógica para la Innovación y dos categorías principales con subsiguientes subcategorías. Seguidamente, se procederá a la descripción hermenéutica correspondiente, la cual se ha denominado como hermenéusis, en coherencia con el Método Etnográfico Interpretativo implementado.

Tabla 3: Sistematización de categorías develadas

\begin{tabular}{c|c}
\hline \multicolumn{2}{c}{ CATEGORÍA MEDULAR: Acción pedagógica para la Innovación } \\
\hline Categorías orientadoras & \multicolumn{1}{c}{ Subcategorías } \\
\hline \multirow{2}{*}{ La innovación en el acto } & 1.1 La comprensión docente sobre la innovación \\
pedagógico de las ETI & 1.2 Incorporación de nuevas ideas \\
& 1.4 Crear ambientes para la innovación \\
& 1.5 Nuevas maneras de Enseñar \\
\hline \multirow{2}{*}{ Pedagogía del aprendizaje } & 2.1 Más allá de enseñar un oficio \\
para la innovación & 2.2 Cambios tangibles \\
& 2.3 Nuevas estrategias \\
\hline
\end{tabular}

Nota: elaboración propia con datos de la investigación.

\section{Hermenéusis de la Categoría orientadora: La innovación en el acto pedagógico de}

\section{las ETI}

Al analizar los relatos de los docentes hacia la innovación en el contexto de aprendizaje de la ETI, asumen que el entendimiento de la innovación en el acto pedagógico constituye un cambio para transformar y mejorar la calidad y pertinencia del aprendizaje hacia la incorporación cotidiana de nuevas ideas que atienda la pertinencia con el entorno y las problemáticas que lo conforman. Por tanto, versionantes relatan sobre la necesidad de generar procesos inter y transdisciplinarios en la escuela y en el currículo, al tiempo de ensayar nuevas formas de enseñar en ambientes acondicionados y adecuados para ello.

Efectivamente, la práctica pedagógica que se lleva a cabo en los talleres y laboratorios 
de la ETI "La Carucieña", requiere la integración de varios conocimientos que son inseparables, ya que el origen de uno depende de otro, es decir, por ejemplo, para realizar una práctica de cordones de soldadura, se debe tener conocimiento básico de electricidad, que permita comprender cómo la energía eléctrica se puede transformar en térmica para fundir el material metálico, logrando así la unión o soldadura de los mismos; es así como estamos en presencia de un verdadero fenómeno multidisciplinar complejo. Se está en presencia de escenarios complejos que se relacionan con la diversidad de pensamientos y van en concordancia con la multidisciplinariedad del conocimiento, sin restarle importancia a las diferentes áreas educativas que influyen en la forma que el docente planifica y realiza las prácticas pedagógicas; esto es el verdadero reto que enfrentan los docentes de la ETI, entender el fenómeno complejo y destacar la necesidad de un enfoque holístico para reorientar el desarrollo del conocimiento educativo estimulado por la Innovación.

A continuación, se aprecia en la tabla 4, la triangulación de las narrativas de los versionantes que otorgan significado a las categorías develadas.

Tabla 4: Sistematización de las narrativas de los versionantes para la categoría orientadora:

La innovación en el acto pedagógico de las ETI

\begin{tabular}{|c|c|c|}
\hline $\begin{array}{l}\text { Categoría } \\
\text { orientadora }\end{array}$ & Subcategorías & Relatos de los versionantes (triangulación de fuentes) \\
\hline \multirow{3}{*}{$\begin{array}{l}\text { La } \\
\text { innovación en el } \\
\text { acto pedagógico de } \\
\text { las ETI }\end{array}$} & $\begin{array}{l}\mathrm{La} \\
\text { comprensión docente } \\
\text { sobre la innovación }\end{array}$ & $\begin{array}{l}\text { (...) Como una actividad donde se busca hacer } \\
\text { la práctica de una forma diferente pero que el } \\
\text { estudiante aprenda mejor y más rápido. EV01L07-08. } \\
\text { (...)Una manera u oportunidad de cambiar y } \\
\text { mejorar cada día el proceso de enseñanza. V03L } 08-08 \text {. }\end{array}$ \\
\hline & $\begin{array}{l}\text { Incorporación } \\
\text { de nuevas ideas }\end{array}$ & $\begin{array}{l}\text { (...)Si claro, con nuevas ideas salen nuevos } \\
\text { pensamientos los cuales se transforman en nuevas } \\
\text { experiencias que se acumulan y se transforman en } \\
\text { nuevos conocimientos para así crear innovación; por eso } \\
\text { los docentes debemos estar claros que debemos formar } \\
\text { ambientes adecuados para la innovación; estos espacios } \\
\text { garantizan la incorporación nuevas ideas de resolver los } \\
\text { problemas, con lo cual estaremos en presencia de } \\
\text { soluciones innovadoras. EV02L15-18. } \\
\text { (...) cuando tú te propones hacer algo diferente, } \\
\text { lo consigues haciendo, pensando y actuando de forma } \\
\text { diferente; todo esto en función de alcanzar un dinámico } \\
\text { camino que nos permita alcanzar las competencias } \\
\text { necesarias que necesitan nuestros estudiantes. EV01L10- } \\
12 \text {. }\end{array}$ \\
\hline & $\begin{array}{l}\text { Integración } \\
\text { curricular de varias } \\
\text { áreas }\end{array}$ & $\begin{array}{l}\text { (...) hay áreas que pueden integrarse de manera } \\
\text { práctica para que existan innovaciones entre ellas en el }\end{array}$ \\
\hline
\end{tabular}




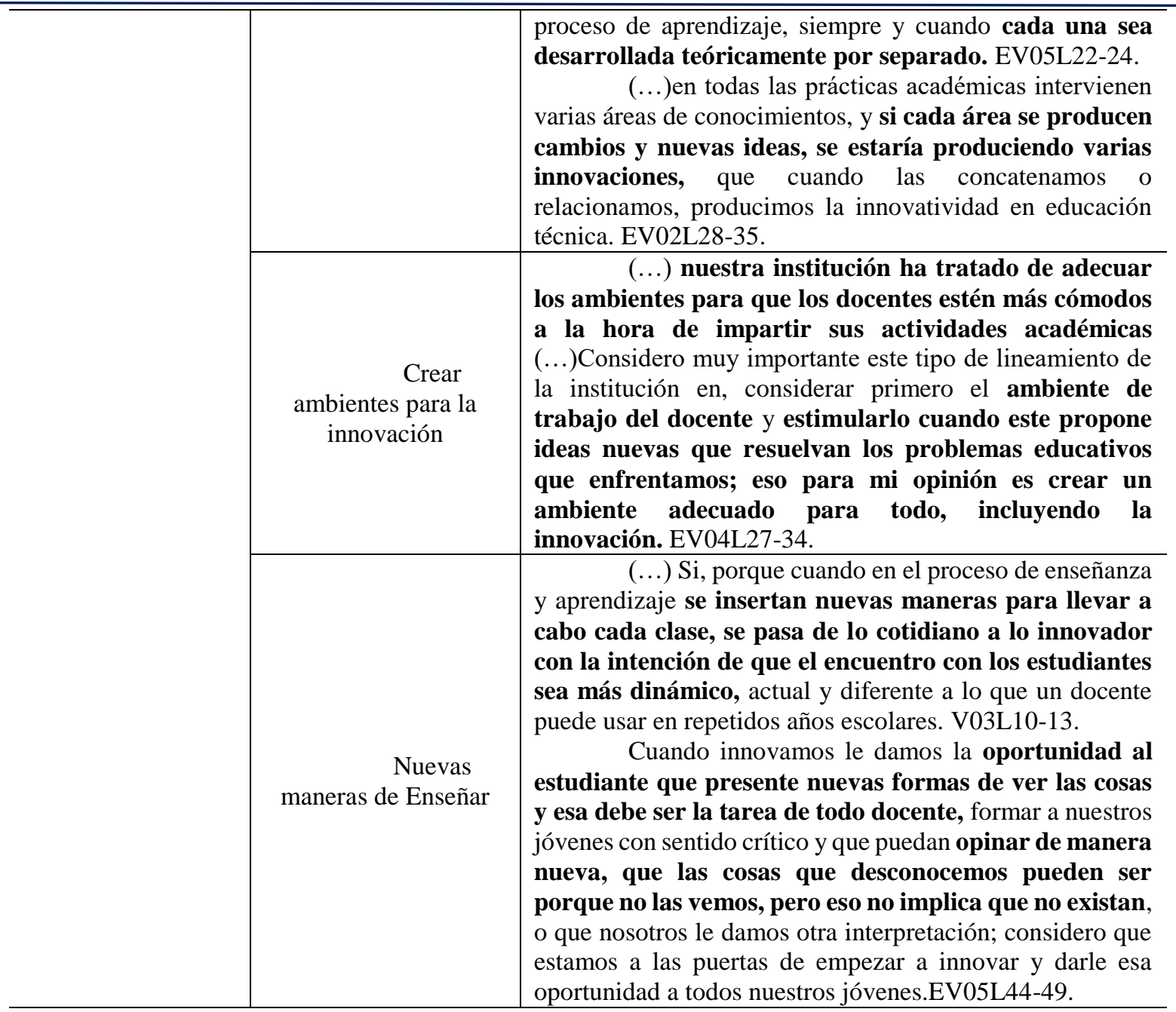

Nota: elaboración propia con datos de la investigación.

\section{Hermenéusis de la Categoría orientadora: Pedagogía del aprendizaje para la} innovación

¿Cómo enseñar y aprender en y para la innovación en un contexto educativo significado por la carencia, pobreza, insuficiencia de recursos educativos y la presencia de equipos tecnológicos de los talleres desactualizados, entre otros? Este es el gran desafío para los docentes de las ETI venezolanas, y de allí la importancia de entender que la innovación implica la alteración del sentido de las prácticas educacionales corrientes y la creación de un nuevo orden; esto supone además asumir una intencionalidad y la movilización de lo que Paulo Freire denominó como "conciencia utópica" (UNESCO, 2016, p.33). Por ello, una pedagogía del aprendizaje para la innovación requiere docentes que más allá de considerarse actores que ejecutan el libreto de los programas, de los libros o de los lineamientos 
directivos, se asuman como verdaderos autores del cambio educativo.

Tal y como se aprecia en la tabla 5, los testimonios de los docentes entrevistados significan que enseñar un oficio para un empleo, como principio ontológico de la formación técnica industrial ya no es suficiente, pues la incertidumbre del contexto socio económico venezolano vinculado al aumento del desempleo, refuerza la necesidad de procurar en el estudiante sus fortalezas, capacidades y competencias desde una perspectiva de la transformación del SER, hacia la sensibilidad de ser actores protagonistas de mejorar y cambiarse a sí mismos para de esta manera cambiar su entorno social.

Por eso es tan importante el atrevimiento hacia el uso de nuevas y diferentes estrategias, en aras de generar conocimientos que atiendan la diversidad de problemas desde la promoción del trabajo en equipo, la colaboración, el diálogo y sobre todo la participación de los estudiantes. Ello en razón que, según la UNESCO, en la medida que un grupo de docentes es capaz de explicitar su trabajo, hacerlo público, ponerlo a discusión y enriquecerlo con las aportaciones de otros colegas, también aumenta su aptitud para mantener e incrementar su proceso innovador (2016).

Tabla 5: Sistematización de las narrativas de los versionantes para la categoría orientadora: Pedagogía del aprendizaje para la innovación

\begin{tabular}{|c|c|c|}
\hline $\begin{array}{l}\text { Categoría } \\
\text { orientadora }\end{array}$ & Subcategorías & Relatos de los "versionantes" (triangulación de fuentes) \\
\hline \multirow{2}{*}{$\begin{array}{l}\text { Pedagogía } \\
\text { del } \\
\text { aprendizaje } \\
\text { para la } \\
\text { innovación }\end{array}$} & $\begin{array}{l}\text { Más allá de enseñar un } \\
\text { oficio }\end{array}$ & $\begin{array}{l}\text { (...)Nuestra institución es una escuela técnica que, durante el } \\
\text { proceso de aprendizaje se le brinda al estudiante una oportunidad } \\
\text { de explorar y descubrir sus fortalezas a nivel vocacional, para que } \\
\text { luego puedan fortalecer estas habilidades durante toda su } \\
\text { permanencia. EV04L42-44. } \\
\text { Nuestra misión es formar al estudiante con los } \\
\text { conocimientos básicos, adiestramiento e instrucción que le pueda } \\
\text { dar un sustento económico y que lo ayude a enfrentarse a las } \\
\text { exigencias laborales que lo rodean, para esto se debe contar con la } \\
\text { participación de todos los involucrados en el proceso educativo. } \\
\text { EV02L51-54. }\end{array}$ \\
\hline & Integralidad del cambio & $\begin{array}{l}\text { (...) Si siempre hay cambios en los procesos de aprendizajes, } \\
\text { recordemos que, igual que los seres humanos todos son diferentes, por } \\
\text { lo tanto, todos aprenden de unas formas diferentes; eso se traduce en } \\
\text { que deben existir diferentes procesos de aprendizaje donde el docente } \\
\text { es el encargado principal de buscar estrategias y métodos diferentes } \\
\text { que produzcan cambios que mejoren este proceso. EV05L56-58. } \\
\text { (...) Si mientras ellos aumentan su nivel de conocimiento } \\
\text { cambian sus aspectos en lo interno son más centrados y se interesan } \\
\text { más por adquirir experiencia para aportar ideas nuevas; en lo } \\
\text { externo sí que pueden ser tangibles como el cabello, modo de vestir, } \\
\text { altura, entre otros. EV03L46-49. }\end{array}$ \\
\hline
\end{tabular}




\begin{tabular}{l|l|l}
\hline \hline Nuevas estrategias & $\begin{array}{c}\text { (...)Las nuevas estrategias se ven dificultadas por la } \\
\text { resistencia a los cambios de muchos docentes, no siendo el caso de } \\
\text { otros docentes que, empujados por su mística educativa, implementan } \\
\text { aisladamente estrategias no monitoreadas y valoradas que permiten un } \\
\text { crecimiento significativo en los participantes. EV01L35-37. } \\
(\ldots) \text { Indudablemente, una de las más indispensables son las } \\
\text { estrategias tecnológicas que ayudan tanto al docente como a los } \\
\text { estudiantes a realizar un proceso de enseñanza y aprendizaje con } \\
\text { innovaciones actuales. EV03L51-55. } \\
\text { Cada docente debe tener muy claro la necesidad de ajustarse a }\end{array}$ \\
$\begin{array}{l}\text { la realidad de su entorno y tener presente las características de sus } \\
\text { estudiantes y los recursos con lo que dispone, aś podrá crear nuevas } \\
\text { estrategias que estén acorde a las exigencias educativas y resolver los } \\
\text { problemas que su entorno le ofrece. EV02L60-65. }\end{array}$ \\
\hline
\end{tabular}

Nota: Elaboración propia con datos de la investigación.

Al revisar los registros de la observación participante, se pudo apreciar el acto pedagógico de un docente identificado como V02MO, quien en una clase de soldadura realiza una estrategia innovadora para introducir a los estudiantes en el aprendizaje de la "soldadura". Ante la inexistencia de recursos simuladores para esta peligrosa competencia de aprendizaje, los estudiantes (sin colocarse la careta para soldar), pasan a los cubículos donde se encuentran los equipos y la máquina para soldar; allí proceden a conectar la pinza de tierra al mesón y en la otra pinza donde se coloca el electrodo o varilla, lo sustituyen por un lápiz de grafito; sobre el mesón colocan un cuaderno y en una hoja empiezan a realizar los diferentes tipos de cordones de acuerdo a lo estipulado en la hoja de actividades pedagógicas para la realización de la práctica. (ROP1).

Esta actividad les permite ejercitar los variados movimientos que se emplean para la elaboración de los diferentes cordones. Seguidamente proceden a colocarse la careta (con la que no tienen visibilidad alguna), y en otra hoja vuelven a realizar la misma operación práctica (ROP2).Una vez terminado, se procede a comparar el diseño escrito de simulación de fabricación de los diferentes tipos de cordones realizados en las dos etapas, a los fines de constatar la alineación lo más perfecta posible de los cordones dibujados, con lo cual el estudiante no solo gana confianza y memoria, sino que ejercita su competencia en forma segura. Seguidamente, pasan al área de trabajo donde realizan el mismo diseño, pero con los instrumentos de soldadura en conexión (ROP3 y ROP4). Ver la figura 1 donde se muestran las imágenes de ROP1 al 4. 
Esta experiencia observada representa una estrategia didáctica generada e implementada por el docente, ante una situación que a todas luces parecía limitante del proceso de aprendizaje, como fue no disponer de los equipos y recursos adecuados. $\mathrm{Su}$ disposición a lograr en sus alumnos las prácticas pedagógicas vinculadas a sus competencias técnico operativas de la educación industrial, permitió elegir, planificar y desarrollar creativamente con los recursos existentes una experiencia de aprendizaje satisfactoria, lo cual indudablemente será el reflejo de una actitud retadora pero también de atrevimiento hacia la innovación, toda vez que "un docente innovador es capaz de transformarse y hacer que los estudiantes participen de forma creativa en sus aulas de clases" (RojasCarrasco, 2019; p. 64).

Figura 1: Secuencia de Imágenes de una clase de soldadura donde el docente ejecuta una estrategia innovadora para introducir a los estudiantes en el aprendizaje de la "soldadura".
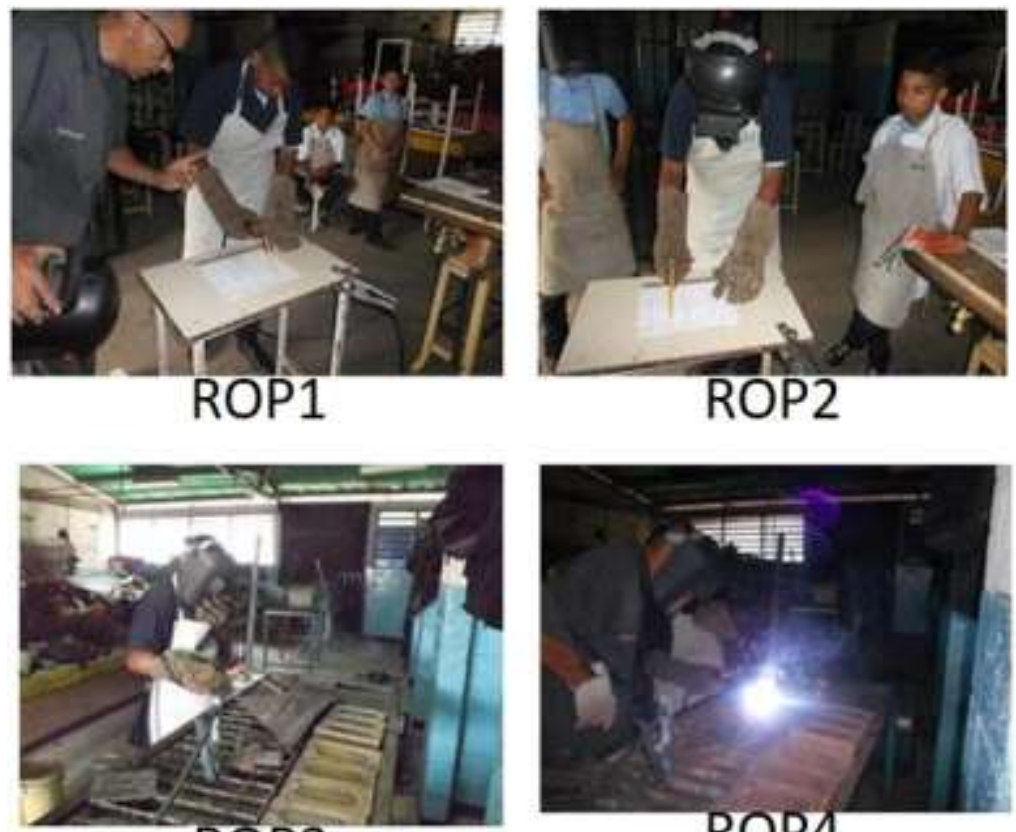

ROP3

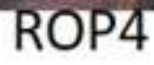

Nota: Elaboración propia con datos de la investigación.

\section{Reflexiones finales}

No queda duda que una educación orientada hacia la innovación representa uno de los retos más altos de la sociedad del siglo XXI, a los fines de apuntar urgentemente a los macro objetivos de desarrollo sustentable de la agenda 2030. La demanda de conocimientos, 
la capacitación laboral y el uso de las tecnologías no serán suficientes si los ciudadanos no se preparan para la creación, el emprendimiento, la transformación y el cambio de sí mismos y de un entorno que permita la convivencia y el bienestar de todos.

En este escenario, la educación técnica en países en desarrollo como Venezuela, se presenta como un espacio de primer orden para la transformación efectiva mediante la preparación de la persona para desenvolverse en un mundo productivo, donde la creatividad y el talento humano interesan más que las materias primas y el perfilamiento de empleos laborales específicos. De allí que la clave para educar para la innovación, es ser innovador, en tanto la UNESCO (2016) refiere que "La innovación no es tanto un producto sino un proceso y una actitud" (p.34), pues hay la finalidad de "desarrollar las competencias de innovación de los estudiantes, que son los resultados de aprendizaje que se refieren a conocimientos, habilidades y actitudes necesarias para que las actividades de innovación tengan éxito" (Kairisto; et.al., 2012).

La experiencia etnográfica reconocida por los docentes de la Escuela Técnica Industrial "La Carucieña", nos perfila que la esencia del ser innovador en el acto pedagógico es el docente, pues sus estudiantes serán el reflejo de la actitud hacia la necesidad de "mejorar", de incorporar cambios y de la dedicación a sobrepasar la educación de la rutina y la inercia. Pues a decir, Iglesias et.al. (2018):

Los docentes son quienes pueden elegir la opción más correcta y beneficiosa para su alumnado y aunque encuentren dificultades y barreras en el camino, han de hacerles frente para poder demostrar que ese cambio o innovación que ha llevado a cabo ha funcionado y dado los resultados queridos o no. Porque si nadie se sale del camino establecido, cambia e innova la educación no avanza con la sociedad. (p.16).

En este sentido, consideramos como necesario, que en el ámbito la educación técnica industrial se generen los espacios reflexivos que permitan apuntar las políticas y estrategias curriculares que conlleven a una educación menos centrada en la repetición de conocimientos teóricos y más orientados a la formación de competencias para la innovación. Se requiere pues la constitución de una cultura escolar para enseñar a innovar e innovar aprendiendo, donde se promueva y reconozca los aportes de los estudiantes y docentes, favoreciendo al tiempo, la autonomía y apertura para experimentar y errar, pues a decir de Martín-Gordillo y Castro-Martínez (2014)'Unos docentes innovadores y una organización que promueva la 
innovación son, por tanto, las condiciones para hacer posible lo más importante: que las competencias innovadoras ocupen un lugar central en la propia formación de los niños y jóvenes" (p.18)

De allí la importancia de "visibilizar" experiencias como la presentada en este artículo, toda vez que los cambios y las transformaciones para una acción pedagógica que fomente la innovación es posible cuando los docentes asumen la voluntad, la intencionalidad y movilización de la "conciencia utópica" planteada por Freire (1997), es decir, cuando en el docente hay una autoconciencia de SER AUTORES creadores y emancipadores, se aleja del ser actor reproductor de una pedagogía sustentada en el conformismo y la pasividad. Solo así, es posible que educación técnica industrial se presente como un camino de esperanza para los muchos estudiantes venezolanos de pocos recursos, quienes eligen sobrepasar las barreras de la pobreza a través de la educación para y en el trabajo.

\section{Referencias}

Antiche,J. (2012) El laboratorio-taller de mecánica automotriz como espacio de aprendizaje en las escuelas técnicas. Revista Educare UPEL IPB.16. (1) 71-97. https://revistas.investigacion-upelipb.com/index.php/educare/article/view/197

Asamblea Nacional de la República Bolivariana de Venezuela. (2009). Ley Orgánica de Educación. Venezuela: Ministerio del Poder Popular para la Comunicación y la Información.

Blanco, R. (2005). Versión preliminar del Módulo "La escuela como centro de la innovación educativa”. UNESCO

Cobo, C. (2016) La Innovación Pendiente, Reflexiones (y Provocaciones) sobre educación, tecnología y conocimiento. Montevideo, Uruguay: Colección Fundación Ceibal.https://revistas.unc.edu.ar/index.php/vesc/article/view/16236

Cuello, P. (2006). La Educación técnica industrial y para el trabajo y las exigencias del mercado laboral. Revista Investigación y Postgrado, 21(1), 1148.http://ve.scielo.org/scielo.php?script=sci_arttext\&pid=S1316$\underline{00872006000100002}$

Drucker, P. (2000).Business Technology.En M. Mejía (Ponente),II Encuentro de Experiencias Educativas de Innovación: La integración curricular una propuesta de 
transformación educativa” Bucaramanga, Colombia: Fe y Alegría - Universidad AutónomaBucaramanga.http://centroderecursos.alboan.org/ebooks/0000/0705/6_Fy A_INT.pdf

Fundación Codespa. (2014). Se celebra la edición 2014 de la Cumbre Mundial para la Innovación en Educación (WISE). https://www.codespa.org/blog/2014/11/14/secelebra-la-edicion-2014-de-la-cumbre-mundial-para-la-innovacion-en-educacionwise/

Freire, P. (1997).Pedagogía de la autonomía. Buenos Aires. Ediciones Siglo XXI.

Geertz, C. (1992). La interpretación de las culturas. Barcelona, España: Gedisa.

Ibarrola, M. (2010). Dilemas de una nueva prioridad a la educación técnico profesional en América Latina, un debate necesario. La educ@ción Revista digital, (144), 129.http://www.educoea.org/portal/La_Educacion_Digital/144/articles/mariadeibarro la.pdf

Iglesias, M.; Lozano, I. \& Roldán, I. (2018). La calidad e innovación educativa en la formación continua docente: un estudio cualitativo en dos centros educativos. Revista Iberoamericana de Educación, 77(1), 13-34.https://doi.org/10.35362/rie7713090

Kairisto, L.; Räsänen, M.; Lehtonen, J. \& Lappalainen, H. (2012). Pedagogía de la Innovación - aprendiendo con métodos activos multidisciplinares. Revista de Docencia Universitaria, 10(1),67-86.https://doi.org/10.4995/redu.2012.6122

Maldonado-Fuentes, A. \& Rodríguez-Alveal, F.(2016). Innovación en los procesos de enseñanza-aprendizaje: Un estudio de casos con la enseñanza justo a tiempo y la instrucción entre pares. Revista Electrónica Educare,20(2), 1-21. http://dx.doi.org/10.15359/ree.20-2.14

Martín-Gordillo, M.\& Castro-Martínez, E. (2014). Educar para innovar, innovar para educar. En D. Pulfer (Director OEI), Congreso Iberoamericano de Ciencia, Tecnología, Innovación y Educación. Universidad de Buenos Aires, Argentina.https://www.researchgate.net/publication/280597980_Educar_para_innov ar_innovar_para_educar

Mogollón, L. (2015). Curso Innovación Educativa y sistematización reflexiva de buenas prácticas pedagógicas.

Módulo

1.Lima.UNESCO.https://es.slideshare.net/paulo910/modulo-1-innovacion 
Naciones Unidas. (2018). La Agenda 2030 y los Objetivos de Desarrollo Sostenible: una oportunidad para América Latina y el Caribe. Santiago de Chile, Chile: Naciones Unidas.CEPAL.https://www.cepal.org/es/publicaciones/40155-la-agenda-2030objetivos-desarrollo-sostenible-oportunidad-america-latina-caribe

Organización Internacional del Trabajo. (2001). Modernización de la formación profesional en América Latina y el Caribe.Montevideo, Uruguay: CINTERFOR.https://www.oei.es/historico/etp/modernizacion_formacion_profes ional_AL_cinterfor.pdf

Piñero,M.; Rivera,M. \& Esteban, E. (2020).El proceder del investigador cualitativo. Precisiones para el proceso de investigación. Lima, Perú: Ediciones de la UNHEVAL-UPEL-IPB. https://doi.org/10.46498/upelipb.lib.0001

Rojas-Carrasco,O. (2019) El rol del maestro en los procesos de innovación educativa. Revista Scientific. 4 (Especial) 54-77. https://doi.org/10.29394/Scientific.issn.25422987.2019.4.E.3.54-67

Ramírez, A. \& León, A. (2012).La enseñanza en la educación media técnica. Anuario del Doctorado en Educación: Pensar la educación, Numero 6, pp. 32-57. http://erevistas.saber.ula.ve/index.php/anuariodoctoradoeducacion/article/view/4357

Robalino, M. \& Eroles, D. (2010). Nuevos tiempos, nuevos desafíos: calidad de la Educación con enfoque de derecho e innovaciones educativas. Oficina de UNESCO, Quito y Red Innovemos (OREALC/UNESCO). Presentación para el Encuentro "Educación e Innovación 2010", organizado por el Ministerio de Educación de Ecuador y VVOB, 2 y 3 de diciembre 2010, Cuenca, Ecuador

Sevilla, M.(2017).Panorama de la educación técnica profesional en América Latina y el Caribe.UNESCO-CEPAL.https://www.cepal.org/es/publicaciones/40920panorama-la-educacion-tecnica-profesional-america-latina-caribe

Simone, J. (1993). Papel de la educación técnico-profesional en el mejoramiento de las capacidades de los trabajadores del sector moderno ante los procesos económicos actuales y los nuevos desarrollos tecnológicos. Revista Iberoamericana de educación, (2), 149-177.https://dialnet.unirioja.es/servlet/articulo?codigo=1019361 
UNESCO. (2015). Educación 2030. Declaración de Incheon y Marco de acción. República de

Corea: Organización de las Naciones Unidas para la Educación, la Ciencia y la Cultura. https://unesdoc.unesco.org/ark:/48223/pf0000245656_spa

UNESCO. (2016).Innovación educativa. Serie "Herramientas de apoyo para el trabajo docente". Texto 1. Lima, Perú: Editora y Comercializadora CARTOLAN E.I.R.L.http://repositorio.minedu.gob.pe/bitstream/handle/MINEDU/5135/Innovaci \%C3\%B3n\%20educativa.pdf? sequence $=1 \&$ is Allowed $=\mathrm{y}$

Velasco, H. y Díaz, A. (2006). La lógica de investigación etnográfica (6ta. ed.). Madrid, España: Editorial Trotto S.A.

Fe y Alegría. (1995). Educación en y para el trabajo liberador y productivo. En XXVI Congreso Internacional. Caracas: Federación Internacional de Fe y Alegría. 